\title{
Detection and quantification of liver fat with MRIT1 dixon sequence in patients with hepatic steatosis and it's comparison with USG
}

\author{
Mathur M. ${ }^{1}$, Sethi G. K. ${ }^{2}$, Mohi J. K. ${ }^{3}$, Kaur N. K. ${ }^{4}$ \\ ${ }^{1}$ Dr. Manoj Mathur, Associate Professor, ${ }^{2}$ Dr. Gaurav Kumar Sethi, Junior Resident, ${ }^{3}$ Dr. Jaswinder Kaur Mohi, \\ Associate Professor, ${ }^{4}$ Dr. Navkiran Kaur, Professor and Head, all authors are affiliated with Department of \\ Radiodiagnosis, Rajindra Hospital, G.M.C. Patiala, Punjab, India
}

Corresponding Author- Dr. Gaurav Kumar Sethi, Junior Resident, Department of Radiodiagnosis, Rajindra Hospital, G.M.C., Patiala, Punjab, India. Email: gauravfaz@gmail.com

\begin{abstract}
Introduction: Hepatic steatosis also known as fatty liver is due to abnormal and excessive accumulation of lipids with in hepatocytes. It is the most common chronic disease of liver. Magnetic resonance techniques can detect fatty liver more accurately than ultrasonography and become primary modalityto assess hepatic steatosis both quantitatively and qualitatively. Aims: The aims of this study were detection and quantification of liver fat by MR technique in patients of hepatic steatosis. Comparison of USG and MRI in detection and quantification of liver fat. Material and Methods: This cross-sectional study was carried out on 50 patients in Department of Radiodiagnosis, Government Medical College, Rajindra Hospital, Patiala in whom risk factors for hepatic steatosis were present. Considering T1 Dixon MRI of liver as reference its comparison with USG was done in these patients for detection of hepatic steatosis and quantification of liver fat. Results: USG had sensitivity $73.68 \%$, specificity $66.67 \%$, positive predictive value $87.50 \%$ and negative predictive value $44.44 \%$ as compared to MRI T1 Dixon sequence for detection of fatty liver. When grading of fatty liver on USG was compared with percentage quantification of liver fat on MRI T1 Dixon sequence of liver, there was considerable ove rlapping of fat percentage in each grade. Conclusion: Advantages of MRI as compared to USG is its more sensitivity and specificity for detection of hepatic steatosis, early detection of hepatc steatosis, quantify liver fat, objective and not observer dependent.
\end{abstract}

Key words: MRI T1 Dixon, Liver fat quantification, Hepatic steatosis

\section{Introduction}

Hepatic steatosis also known as fatty liver is caused by abnormal and excessive accumulation of lipids with in the hepatocytes[1].A liver is considered 'fat' or 'steatotic' when fat-containing vacuoles accumulate in the hepatocytes and the total fat content exceeds $5 \%$ of the net weight of the liver[2]. It is divided mainly into two etiological types - alcoholic hepatic steatosis and non-alcoholic hepatic steatosis also known as nonalcoholic fatty liver disease (NAFLD). Major risk factors for non-alcoholic fatty liver are obesity, type 2 diabetes mellitus and dyslipidemia[3]. Hepatic steatosis can also be secondary to drugs, toxins, chronic hepatitis $\mathrm{C}$, hepatitis B viral infections, storage disorders (e.g., hemochromatosis, Wilson disease, glycogen storage disease), pregnancy[4].

Manuscript received: $05^{\text {th }}$ February 2018

Reviewed: $12^{\text {th }}$ February 2018

Author Corrected: $17^{\text {th }}$ February 2018

Accepted for Publication: $22^{\text {nd }}$ February 2018
USG in patient of hepatic steatosis-Hepatic steatosis results in increased echogenicity (brightness) of the liver parenchyma in comparison with the renal cortex and spleen. This occurs because of the increasing interfaces created in the liver by lipid accumulation, leading to more echoes returning to the transducer, thus making the liver appear bright[5]. In this setting, the visibility of the hepatic vessel walls and the diaphragm is reduced. In patients of hepatic steatosis liver size may increase. Various (0-3) grades of steatosis have been proposed based on visual analysis of the intensity of the echogenicity. When echogenicity of liver is less or equal to right renal cortex, it is grade 0 or non-fatty liver. When the echogenicity is just increased than right renal cortex, it is grade 1 fatty liver (mild). When the echogenic liver obscures the echogenic walls of portal vein branches, it is grade 2 fatty liver (moderate). When the echogenic liver obscures the diaphragmatic outline, it is grade 3 fatty liver (severe)[6]. If disease progress 


\section{Original Research Article}

further to cirrhosis then size may decrease, outline of liver becomes irregular, echotexture becomes heterogenous.

MR evaluation of patient of hepatic steatosis-MR techniques help to detect fat signals on the basis of the difference in precessional frequency between water and fat signals[7]. A key concept is that MR techniques are unique in their ability to decompose the liver signal into its water and fat components. After the liver signal is decomposed, the signal fat-fraction $(\eta)$ is calculated as the signal from fat protons divided by the combined signal from fat and water protons in the liver.

$$
\eta=\frac{F}{W+F}
$$

To calculate of fat signal percentage of liver also known as hepatic fat fraction, signal fat fraction is multiplied by100.[8]Grading of severity of hepatic steatosis done based on liver fat percentage. Liver fat percentage range of $0-5 \%$ as normal, $5.1-15 \%$ as mild grade hepatic steatosis, $15.1-30 \%$ as moderade grade hepaic steatosis and more than $30 \%$ as severe grade hepatic steatosis[9].

Dixon MRI sequence (Chemical Shift Imaging) -It is based on that the difference in processional frequencies between water and fat protons. The difference enables the use of chemical shift techniques to accurately detect and quantify fatty infiltration. With an echo time at which the fat and water signals are in phase, the signals add constructively and when they are out of phase, the signals cancel[10]. Whereas the normal liver parenchyma exhibits similar signal intensity on in-phase and out-of-phase images, fatty liver shows diminished signal intensity on out-of-phase images, with the reduction being more evident in the presence of severe fatty infiltration[11]. Dixon is done on T1 weighted images. In Dixon sequence four sets of different images are formed- in-phase image, out-phase image, fat-only image and water-only image.

Aims of this study weredetection and quantification of liver fat by MR technique in patients of hepatic steatosis. Comparison of USG and MRI in detection and quantification of liver fat.

\section{Material and Methods}

This cross-sectional study was carried out on 50 patient in Department of Radiodiagnosis, Government Medical College, Rajindra Hospital, Patiala which are referred to this department from various other departments for ultrasonography (USG) of abdomen and in whom single or multiple risk factors for hepatic steatosis were present.

\section{Inclusion criteria}

- Patients with single or multiple risk factors for hepatic steatosis in which either diffuse hepatic steatosis or normal liver was detected on ultrasonography of abdomen.

- Patients giving consent for MR imaging and are willing to enroll in study.

\section{Exclusion criteria}

- Patient having cardiac pacemaker, electromagnetic implant.

- Patient not giving consent.

\section{Equipment}

- USG by Philips HD 11 machine

- MR techniques by 1.5-T superconductive scanner (Siemens1.5T Magnetomaera MRI machine).

Ultrasonography technique- USG was performed with philips HD 11 machine. Patients were examined in supine position. An appropriate transducer frequency ranging from 2.5 to $5 \mathrm{MHz}$ had been used, depending on the body habitus. Both curvilinear and linear probes were used. Sagittal, transverse and obliques views were taken and images of liver, right kidney and others required images were taken.

MRI technique- Patients were examined in supine position with proper positioning. All the studies were performed by 1.5-T superconductive scanner (Siemens 1.5T MagnetomAera MRI machine), flex (abdomen coil) was used.Localiser images in axial, coronal and sagittal planes were obtained.

T1-weighted Dixon gradient echoes images $(6.69 \mathrm{~ms}$ repetition time, $2.39 \mathrm{~ms}$ echo time $1,4.77 \mathrm{~ms}$ echo time 2) were obtained in the axial planes.Out-of-phase images at TE $1=2.39 \mathrm{~ms}$ and In-phase images at TE 2 $=4.77 \mathrm{~ms}$. Dixon images were obtained in the axial plane with $3 \mathrm{~mm}$ slice thickness, FOV (field of view) $380 \mathrm{~mm}$ and with flip angle of 10 degree.

Image analysis (method for calculation of percentage of liver fat in T1 weighted Dixon sequence)-In Dixon sequence four sets of different images are formed- inphase image, out-phase image, fat-only image and water-only image. 


\section{Original Research Article}

To estimate the signal fat-fraction, we assume (a) the signal intensity from fat is less than the signal intensity from water (i.e.,SFat $\leq$ SWater), (b) the signal intensity from OP images represents the difference in water and fat signals (i.e., $S O P=S$ Water $-S F a t$ ), and (c) the signal intensity from IP images represents the sum of water and fat signals (i.e., SIP $=$ SWater + SFat). Re-arranging terms in (b) and (c), and assuming (a), the MR signal can be decomposed into its water and fat components based on arithmetic combinations of the in-phase and out-of-phase signal intensities:

$$
\begin{aligned}
& S_{\text {Water }}=\left|S_{I P}+S_{O P}\right| \\
& S_{\text {Fat }}=\left|S_{I P}-S_{O P}\right|
\end{aligned}
$$

Inserting the SFat and SWater terms into previous equation generates new equation which permits calculation of the signal fat-fraction from the out-ofphase and in-phase signal intensities.

$$
\eta=\frac{\left|S_{I P}-S_{O P}\right|}{2 * S_{I P}}
$$

For calculation of $\%$ of liver fat, signal fat fraction is multiplied by 100 .

For quantification of liver fat by Dixon technique spleen is taken as reference. For calculation of Sip or Sop, images are taken in which both liver and spleen are visible. A small circle of roi (region of interest) is applied on right lobe of liver and similar roiapplied on spleen both on similar axial section image on in-phase and out-phase image. Area of ROI should be more than $1 \mathrm{~cm}$ square and it should be away from major vessels. Sip is calculated by dividing signal intensity of liver by signal intensity of spleen on in-phase image and Sop is calculated by dividing signal intensity of liver by signal intensity of spleen on out- phase image.

Study analysis- The sample study was limited to 50 patients. Informed consent will be taken from all the subjects before starting the study. Patients with single or multiple risk factors for hepatic steatosis who referred from other departments for USG abdomen in which either diffuse hepatic steatosisor normal liver was detected on USG were taken in this study. After fulfillment of all the inclusion and exclusion criterias, MRI of liver was done in these cases. MRI sequences done was T1 weighted Dixon sequence (chemical shift imaging)for detection hepatic steatosis and quantification of liver fat. Considering T1 Dixon MRI of liver as reference its comparison with USG was done in these patients. Statistical analysis of comparison of USG and MRI done. Prevalence of hepatic steatosis and its clinical features were assessed in these patients.

\section{Results}

Out of 50 patients included in this study, 33 were females and 17 were males. In our study out of 50 patients, fatty liver was seen in 38 patients by MRI T1 Dixon sequence of liver (overall prevalence $76 \%$ ). Out of these 38 patients of fatty liver, 24 were females and 14 were males, with female to male ratio 1.71:1. Overall mean age of patients of fatty liver was $47.13 \pm 11.24$.

Out of 13 patients with alcoholic as a risk factor, fatty liver was seen in 10 patients (76\%). Out of 33 patients with obesity as a risk factor, fatty liver was seen in 27 patients $(81.82 \%)$.Out of 16 patients with diabetes mellitus as a risk factor, fatty liver was seen in 12 patients $(75 \%)$.Out of 15 patients with dyslipidemia as a risk factor, fatty liver was seen in 12 patients $(80 \%)$.Fatty liver was seen in all the 8 patients $(100 \%)$ with hepatotoxic drug (tamoxifen) therapy as a risk factor. Out of 3 patients with hepatitis $\mathrm{C}$ as a risk factor, fatty liver was seen in 1 patient (33.33\%). These risk factors were statistically significant for fatty liver ( $\mathrm{p}$ value is 0.026 which is $<0.05$ ).

Out of 38 patients of fatty liver, 20 patients (52.63\%) were asymptomatic, 11 patients $(28.95 \%)$ presented with chief complaint of generalized weakness, 3 patients $(7.89 \%)$ presented with dyspepsia, 3 patients $(7.89 \%)$ presented with pain abdomen and 1 patient $(2.63 \%)$ presented with abdominal distension.

Out of 50 patients included in this study, USG detected fatty liver in 32 patients (64\%), in rest of the 18 patients (36\%) no fatty liver was detected. Grading of fatty liver in these patients done by USG showed 18 patients (36\%) had non-fatty liver, 11 patients $(22 \%)$ had mild (grade 1$)$ fatty liver, 14 patients $(28 \%)$ had moderate (grade2) fatty liver and 7 patients $(14 \%)$ had severe (grade 3) fatty liver. Out of 50 patients, hepatomegaly was seen in 29 patients $(58 \%)$ and no hepatomegaly was seen in 21 patients (42\%). Irregular liver shape and ascites was seen in 3 patients $(6 \%)$.Gall bladder stones were seen in 5 patients $(10 \%)$. 
Table-1: USG abdomen findings of patients included in study

\begin{tabular}{|c|c|c|c|}
\hline USG liver findings & Present/Absent & No. of patients & Percentage \\
\hline \multirow{2}{*}{ Fatty liver } & Yes & 32 & $64 \%$ \\
\cline { 2 - 4 } & No & 18 & $36 \%$ \\
\hline \multirow{3}{*}{ Grading of fatty liver } & Non-fatty liver (0) & 18 & $36 \%$ \\
\cline { 2 - 4 } & Mild (1) & 11 & $22 \%$ \\
\cline { 2 - 4 } & Moderate (2) & 14 & $28 \%$ \\
\hline \multirow{2}{*}{ Hepatomegaly } & Sever (3) & 29 & $14 \%$ \\
\cline { 2 - 4 } & Yes & 21 & $58 \%$ \\
\hline \multirow{2}{*}{ Other associated findings } & Irregular liver shape \& ascites & 3 & $42 \%$ \\
\cline { 2 - 4 } & Gall bladder stone & 5 & $6 \%$ \\
\hline
\end{tabular}

Table-2: MRI T1 Dixon sequence of liver for detection of fatty liver and quantification of liver fat in patients included in this study

\begin{tabular}{|c|c|c|c|}
\hline MRI T1 Dixon sequence of liver & Present/Absent & No. of patients & Percentage \\
\hline \multirow{2}{*}{ Fatty liver detected } & Yes & 38 & $66 \%$ \\
\hline & No & 12 & $24 \%$ \\
\hline \multirow{4}{*}{ Quantification of liver fat } & $0-5 \%$ Fat (non-fatty liver) & 12 & $24 \%$ \\
\hline & $\begin{array}{c}5.1-15 \% \text { Fat } \\
\text { (mild grade fatty liver) }\end{array}$ & 12 & $24 \%$ \\
\hline & $\begin{array}{c}15.1-30 \% \text { Fat } \\
\text { (moderate grade fatty liver) }\end{array}$ & 14 & $28 \%$ \\
\hline & $\begin{array}{c}>30 \% \text { Fat } \\
\text { (severe grade fatty liver) }\end{array}$ & 12 & $24 \%$ \\
\hline
\end{tabular}

Out of 50 patients included in our study, MRI T1 Dixon sequence of liver detected fatty liver in 38 patients (76\%), in rest of the 12 patients $(24 \%)$ no fatty liver was detected. Quantification of liver fat done by MRI T1 Dixon sequence showed 12 patients $(24 \%$ ) had liver fat in the range of $0-5 \%$ (non fatty liver), 12 patients (24\%) had liver fat in the range of 5.1-15\% (mild grade fatty liver), 14 patients (28\%) had liver fat in the range in 15.1-30\% (moderate grade fatty liver)and 12 patients $(24 \%$ ) had liver fat $>30 \%$ (severe grade fatty liver). Hepatic fat fraction in our study ranged from $1.2 \%-43.1 \%$. Comparison of USG abdomen with MRI T1 dixon sequence of liver was done by considering MRI T1 dixon sequence as reference. Out of 38 patients of fatty liver detected by MRI T1 Dixon sequence of liver, 28 patients had fatty liver and 10 patients had non-fatty liver on USG abdomen. Out of 12 patients diagnosed as non-fatty liver by MRI T1 Dixon sequence of liver, 8 patients had non-fatty liver and 4 patients had fatty liver by USG abdomen.

Table-3: Comparison of USG to detect fatty liver with percentage of liver fat detected by MRI T1 Dixon sequence of liver

\begin{tabular}{|c|c|c|c|c|c|}
\hline $\begin{array}{c}\text { Quantification of liver fat } \\
\text { by MRI T1 Dixon sequence } \\
\text { of liver }\end{array}$ & $\begin{array}{c}\text { No. of } \\
\text { Patients }\end{array}$ & $\begin{array}{c}\text { Fatty liver } \\
\text { cases detected } \\
\text { by USG }\end{array}$ & $\begin{array}{c}\text { \%age of fatty liver } \\
\text { cases detected by } \\
\text { USG }\end{array}$ & $\chi^{\mathbf{2}}$ & P value \\
\hline $\begin{array}{c}5.1-15 \% \text { fat (mild grade fatty } \\
\text { liver) }\end{array}$ & 12 & 5 & $41.67 \%$ & 2.12 & $0.146(\mathrm{NS})$ \\
\hline $\begin{array}{c}15.1-30 \% \text { fat (moderate grade } \\
\text { fatty liver) }\end{array}$ & 14 & 11 & $78.57 \%$ & 21.11 & $0.006(\mathrm{~S})$ \\
\hline $\begin{array}{c}>30 \% \text { fat (severe grade fatty } \\
\text { liver) }\end{array}$ & 12 & 12 & $100 \%$ & 20.17 & 0.001 \\
$(\mathrm{HS})$
\end{tabular}

Out of 12 patients with liver fat range 5.1- 15\% (mild grade fatty liver) by MRI T1 Dixon sequence of liver, USG abdomen detected fatty liver in 5 cases (41.67\%). Out of 14 patients with liver fat range 15.1-30\% (moderate grade fatty liver) by MRI T1 Dixon sequence of liver, USG abdomen detected 11 cases (78.57\%). USG abdomen detected fatty liver in all of 12 patients (100\%) which had liver fat $>30 \%$ (severe grade fatty liver) by MRI T1 Dixon sequence of liver. So in 


\section{Original Research Article}

our study USG abdomen was highly significant (p-value of 0.001 ) in detection of fatty liver which was severe grade on MRI. USG abdomen was significant (p-value 0.006) in detection of fatty liver which was moderate grade on MRI and not significant (P value 0.146) in detection of fatty liver which was mild grade on MRI. Comparison of USG grading of fatty liver with quantification of liver fat by MRI T1 dixon sequence was done. Grade 0 (non-fatty liver) patients on USG abdomen had liver fat $1.2-18.4 \%$ on MRI T1 Dixon sequence of liver. Grade 1 (mild fatty liver) patients on USG abdomen had liver fat $2.1-26 \%$ on MRI T1 Dixon sequence of liver. Grade 2 (moderate fatty liver) patients on USG abdomen had liver fat $2.7-37.3 \%$ on MRI T1 Dixon sequence of liver. Grade 3 (severe fatty liver) patients on USG abdomen had liver fat $14.3-43.1 \%$ on MRI T1 Dixon sequence of liver.

Images of 30 years old male who is alcoholic and has dyslipidemia

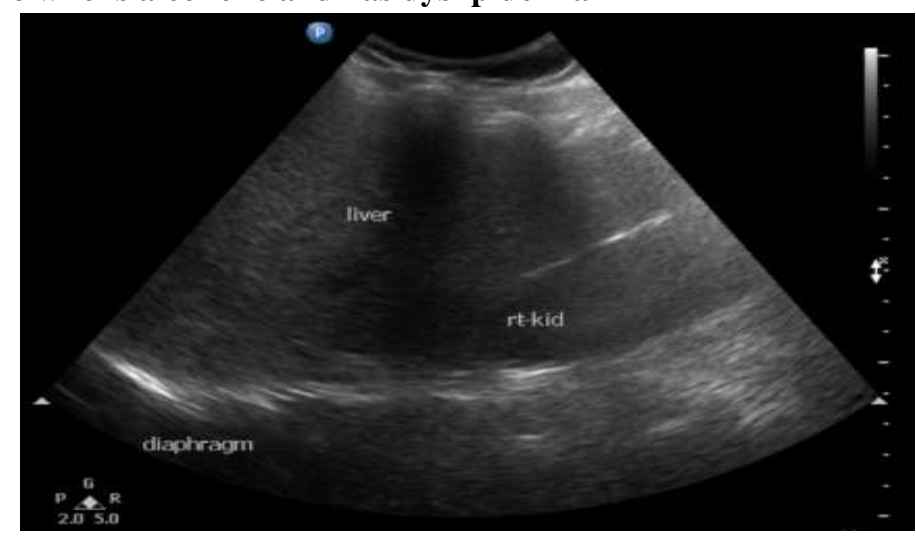

Figure- A

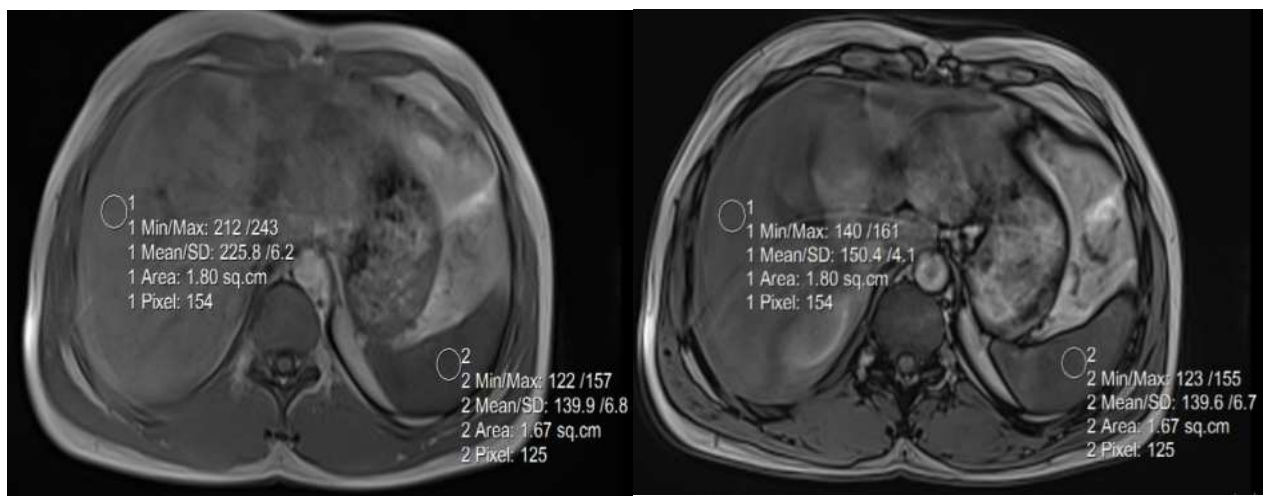

Figure $B$ and figure $C$

Figure A showing USG abdomen image showing liver has almost similar echopattern as cortex of right kidney suggestive of non fatty liver. Mean signal intensity of liver and spleen on MRI T1 Dixon in-phase image (figure B) are 225.8 and 139.9, and that on out-phase image (figure C) are 150.4 and 139.6. Liver fat percentage in this case is $16.7 \%$ by using methods as described previously. USG results in this case is false negative as compared to MRI for detection Of fatty liver.

Images of 31 years old female who is obese and dyslipedemic

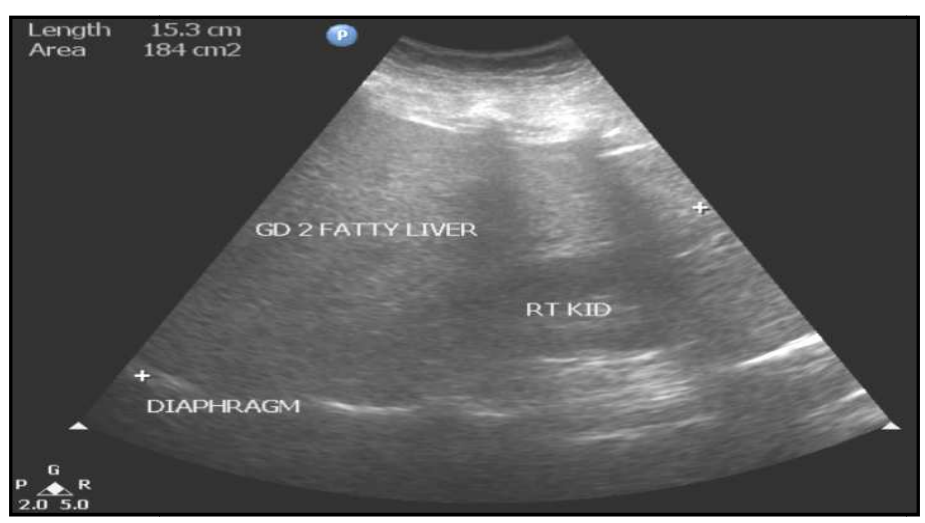




\section{Original Research Article}

USG image - Liver is bright in echopattern as compared to cortex of right kidney with slight obscuration of walls of hepatic vessels and diaphragm, suggestive of grade 2 fatty liver.

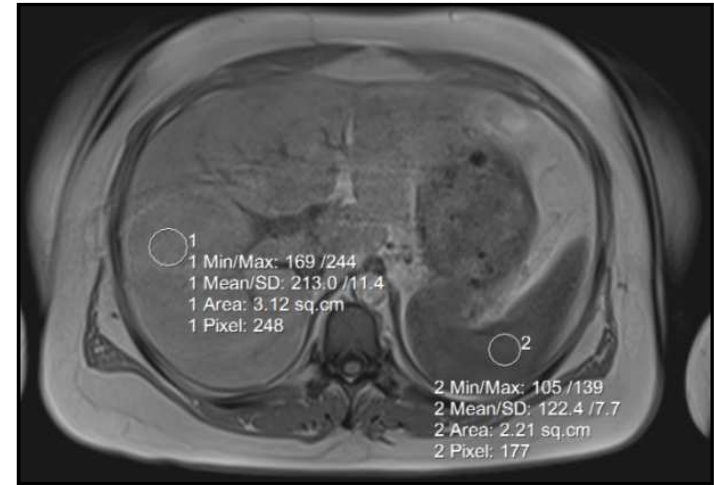

MRI T1 Dixon in-phase image

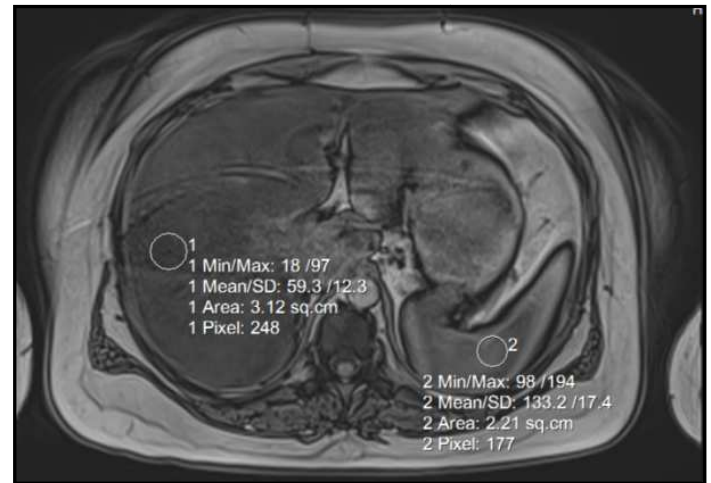

MRI T1 Dixon out-phase image

Mean signal intensity of liver and spleen on in-phase image are 213.0 and 122.4 , and that on out-phase image are 59.3 and 133.2. Liver fat percentage in this case is $37.3 \%$ by using method as described.USG results in this case is true positive as compared to MRI for detection of fatty liver

\section{Discussion}

This cross-sectional study was conducted in The Department of Radiodiagnosis, Rajindra Hospital, G.M.C. Patiala for a period of two years. Fifty patients with single or multiple risk factors for hepatic steatosis who came for USG abdomen in whom either diffuse hepatic steatosisor normal liver was detected on USG were taken in this study. MRI of liver was done in these cases for detection and quantification of liver fat. MRI sequences done was T1 weighted Dixon sequence (chemical shift imaging). Considering T1 Dixon MRI of liver as reference its comparison with USG was done in these patients. Prevalence of hepatic steatosis and its clinical features were assessed in these patients.

In our study out of 50 patients with single or multiple risk factors, fatty liver was seen in 38 patients (overall prevalence $76 \%$ ). Out of these 38 patients 24 were females and 14 were males, with female to male ratio 1.71:1. Overall mean age of patients of fatty liver was $47.13 \pm 11.24$ years. Peak prevalence in females was in 51-60 years age group and in males it was in 31-40 years age group. Similar results were obtained by Cheng et al[12] in 2013, they concluded the prevalence of fatty liver was significantly higher in males than in females prior to age of 50 years, but prevalence was significantly higher in females than in males after the age of 50 years.

In our study fatty liver was seen in $76 \%$ of alcoholics, $81.82 \%$ of obese, $75 \%$ of diabetics, $80 \%$ of dyslipidemics, $100 \%$ in patients on tamoxifen drug therapy and $33.33 \%$ of hepatitis $\mathrm{C}$ patients. In our study obesity was the most common risk factor for fatty liver followed by dyslipidemia, diabetes mellitus, alcoholism, hepatotoxic drug therapy and hepatits $\mathrm{C}$ in decreasing order. Alcoholism as a cause of fatty liver was seen only in males in our study. As number of risk factors for fatty liver increased, prevalence of fatty liver increased. Positive correlation was seen in a study done by Bellentini et al[13] in 2000 in which they found that the prevalence of hepatic steatosis was increased in alcoholics (46.4\%) and in obese persons (75.8\%) compared with controls $(16.4 \%)$. Steatosis was found in $94.5 \%$ in obese alcoholics. Similar findings were seen in our study in which fatty liver prevalence increased by increasing number of risk factors.

In our study mostly, patients of fatty liver were asymptomatic or presented with vague complaints like generalized weakness or dyspepsia. Few patients presented with pain abdomen which can be due to associated problems e.g. gall bladder stones were seen in few patients. One patient presented with abdominal distension, in this patient USG findings were ascites and irregular shape of liver. These findings suggest that this patient might have progressed to liver cirrhosis. Positive correlation was seen in the study done by Wong et al in 2004 in hepatic steatosis patients in which they found that the majority of patients were asymptomatic, $14.7 \%$ patients had generalized malaise and $4.8 \%$ had right upper quadrant pain.

Out of 38 patients who were positive for fatty liver by considering MRI T1 Dixon sequence of liver as reference, hepatomegaly was seen in 27 patients (71.05\%). Hepatomegaly was also seen in 2 out of 12 


\section{Original Research Article}

patients who were negative for fatty liver. So in our study presence of hepatomegaly on USG was significant finding in patients of fatty liver.

USG had sensitivity $73.68 \%$, specificity $66.67 \%$, positive predictive value $87.50 \%$ and negative predictive value $44.44 \%$ as compared to MRI T1 Dixon sequence for detection of fatty liver. USG sensitivity for detection of fatty liver was $41.67 \%, 78.68 \%$ and $100 \%$ for mild, moderate and severe grade of fatty liver on MRI T1 Dixon of liver respectively. Sensitivity of USG was less as compared to MRI in detection of fatty liver because mild form of disease is difficult to detect by USG. Less specificity of USG was because in obese patients due to impaired beam penetration and limited liver visualization, false obscuration of walls of hepatic vessels and diaphragm occur so false positive results occur. Positive correlation was seen in a study done by Palmentieri et al[14] in 2006 in which they found overall sensitivity of USG for detection of hepatic steatosis was $67 \%$.Sensitivity of USG for detecting hepatic steatosis was $91 \%$ in patients who had percentage of liver fat of moderate to severe grade. Similar results were seen in a study done by Pereze et al [15] in 2007, they found that the sensitivity of an USG ranged from $11.4 \%$ to $88.2 \%$ and the specificity ranged from $40.4 \%$ to $86.2 \%$, depending on the degree of steatosis.Hernaez et al [16] in 2011 conducted a metaanalysis and found that Ultrasound had sensitivity range of $60.5-99.3 \%$ and, specificity range of $53.3-93.4 \%$ as compared to MRI. These results show positive correlation to our study.

Grade 0 (non-fatty liver) patients on USG abdomenhad liver fat $1.2-18.4 \%$ on MRI T1 Dixon sequence of liver. Grade 1 (mild fatty liver) patients on USG abdomen had liver fat $2.1-26 \%$ on MRI T1 Dixon sequence of liver. Grade 2 (moderate fatty liver) patients on USG abdomen had liver fat $2.7-37.3 \%$ on MRI T1 Dixon sequence of liver. Grade 3 (severe fatty liver) patients on USG abdomen had liver fat $14.3-43.1 \%$ on MRI T1 Dixon sequence of liver. So when grading of fatty liver on USG was compared with quantification of liver fat on MRI T1 Dixon of liver, there was considerable overlapping of fat percentage in each grade. Many patients with same percentage of liver fat on MRI had different grades of fatty liver on USG. This occurred because grading of fatty liver on USG is subjective and observer dependent while quantification of liver fat on MRI is objective and not observer dependent.Positive correlation was seen in a study done by Pacifico et al [17] in 2007, they used T1 weighed dual echo MRI for fat quantification and compared it with USG grade.
They found that liver fat percentage by MRI ranged 2$37 \%$ in moderate grade hepatic steastosis on USG and it ranged $11-25 \%$ in severe grade hepatic steatosis on USG.

\section{Conclusion}

Hepatic steatosis (fatty liver) disease is most commonly seen in middle age group. Disease has more prevalence in patients who are obese, diabetic, dyslipidemic, alcoholic and in patients on some types of drug therapy.Disease prevalence increases if number of risk factors increase. Patients with hepatic steatosis are usually asymptomatic or present with vague complaints like generalised weakness, dyspepsia and pain abdomen. Hepatomegaly on USG imaging is seen in majority of these patient.

Advantages of Magnetic resonance imaging are early detection of hepatic steatosisi.e. its mild form of disease can be detected, quantifies percentage of live fat, objective, not observer dependent, simple, noninvasive, no radiation exposure and no contrast media required. This quantification of liver fat can be used in follow up of patients of hepatic steatosis which are on treatment. Major disadvantages of MRI that it is costly and may not be available in small setup. Advantages of USG over MRI are its low cost and wide availability. Disadvantages of USG as compared to MRI are its low sensitivity specially in mild form of disease, low specificity specially in obese, subjective nature, operator dependent, can not exactly tell severity of disease, cannot quantify liver fat so can not be used in follow up of patients.

\section{Funding: Nil, Conflict of interest: None. Permission of IRB: Yes}

\section{Bibliography}

1. Fromenty B, Pessayre D. Inhibition of mitochondrial beta-oxidation as a mechanism of hepatotoxicity. PharmacolTher. 1995;67(1):101-54.

2. Lebovics E and Rubin J. Non-alcoholic fatty liver disease (NAFLD): why you should care, when you should worry, what you should do. Diabetes Metab Res Rev. 2011;27(5):419-24.

3. Parekh S, Anania FA. Abnormallipid and glucosemetabolism in obesity: implications for nonalcoholic fatty liver disease. Gastroenterology. 2007 May;132(6):2191-207. 


\section{Original Research Article}

4. Shoelson SE, Herrero L, Naaz A. Obesity, inflammation, and insulin resistance. Gastroenterology. 2007 May;132(6):2169-80.

5. Yajima Y, Ohta K, Narui T, Abe R, Suzuki H, Ohtsuki M. Ultrasonographicaldiagnosis of fatty liver: significance of the liver-kidneycontrast. Tohoku J Exp Med. 1983 Jan;139(1):43-50.

6. Saadeh S, Younossi ZM, Remer EM, Gramlich T, Ong JP, Hurley M, Mullen KD, Cooper JN, Sheridan MJ. The utility of radiologicalimaging in nonalcoholic fatty liver disease. Gastroenterology. 2002 Sep;123(3):745-50.

7. Rofsky NM, Fleishaker H. CT and MRI of diffuseliver disease. Semin Ultrasound CT MR. 1995 Feb;16(1):16-33.

8. Reeder SB, Cruite I, Hamilton G, Sirlin CB. QuantitativeAssessment of LiverFat with Magnetic Resonance Imaging and Spectroscopy. J MagnResonImaging. 2011 Oct;34(4):spcone.

9. Pacifico L, Martino MD, Catalano C, Panebianco V, Bezzi M, Anania C, Chiesa C. T1-weighteddualechoMRI for fatquantification in pediatricnonalcoholic fatty liver disease. World J Gastroenterol. $2011 \mathrm{Jul}$ 7;17(25):3012-9. doi: 10.3748/wjg.v17.i25.3012.

10. Hussain HK, Chenevert TL, Londy FJ, Gulani V, Swanson SD, McKenna BJ, Appelman HD, Adusumilli S, Greenson JK, Conjeevaram HS. Hepaticfatfraction: MRimaging for quantitativemeasurement and display-early experience.Radiology. 2005 Dec;237(3):1048-55. Epub 2005 Oct 19.

11. Kreft BP, Tanimoto A, Baba Y, Zhao L, Chen J, Middleton MS, Compton CC, Finn JP, Stark DD.
Diagnosis of fatty liver with MR imaging. J MagnReson Imaging. 1992 Jul-Aug;2(4):463-71

12. Cheng HY, Wang HY, Chang WH, Lin SC, Chu $\mathrm{CH}$, Wang TE et al. Nonalcoholic fatty liver disease: prevalence, influence on age and sex, and relationship with metabolic syndrome and insulin resistance. International Journal of Gerontology. 2013 Dec 31;7(4):194-8.

13. Bellentani S, Saccoccio G, Masutti F, Crocè LS, Brandi G, Sasso F et al. Prevalence of and risk factors for hepatic steatosis in Northern Italy. Ann Intern Med. 2000;132(2):112-7.

14. Palmentieri B, de Sio I, La Mura V, Masarone M, Vecchione R, Bruno S, Torella R, Persico M. The role of bright liver echo pattern on ultrasound B-mode examination in the diagnosis of liver steatosis. Dig Liver Dis. 2006 Jul;38(7):485-9. Epub 2006 May 22.

15. Perez NE, Siddiqui FA, Mutchnick MG, Dhar R, Tobi M, Ullah $\mathrm{N}$ et al. Ultrasound diagnosis of fatty liver in patients with chronic liver disease: a retrospective observational study. J Clin Gastroenterol. 2007;41(6):624-9.

16. Hernaez R, Lazo M, Bonekamp S, Kamel I, Brancati FL, Guallar E, Clark JM. Diagnostic accuracy and reliability of ultrasonography for the detection of fatty liver: A meta-analysis. Hepatology. 2011;54(3): 1082-90.

17. Pacifico L, Celestre M, Anania C, Paolantonio P, Chiesa C, Laghi A. MRI and ultrasound for hepaticfatquantification:relationships to clinical and metaboliccharacteristics of pediatricnonalcoholic fatty liver disease. Acta Paediatr. 2007 Apr;96(4):542-7. Epub 2007 Feb 14.

\section{How to cite this article?}

Mathur M., Sethi G. K., Mohi J. K., Kaur N. K. Detection and quantification of liver fat with MRIT1 dixon sequence in patients with hepatic steatosis and it's comparison with USG. Int J Med Res Rev 2018;6 (02):102-109.doi:10.17511/ijmrr. 2018.i02.06. 